Rev. Elev. Méd. vét. Pays trop., 1974, 27 (2) : 163-168

\title{
Note sur l'écologie de la peste équine africaine
}

\author{
par P. BOURDIN $\left({ }^{*}\right)$ et A. LAURENT $\left({ }^{*}\right)$
}

\begin{abstract}
RESUME
L'analyse des faits et travaux concernant l'écologie de la P.E.A. montre que cette affection sévit à l'état endémique dans les pays à climat tropical sec et périodiquement déborde au Sud et au Nord vers les pays voisins. Le cheval est très sensible au virus mais dans les pays où la P.E.A. est ancienne, il a acquis une immunité naturelle solide. Le mode de transmission s'apparente à celui des arbovirus, deux vecteurs sont incriminés: les moustiques et les culicoïdes. Dans la nature, l'isolement du virus est difficile et n'a été réalisé que chez culicoödes spp. La multiplication du virus chez les deux vecteurs reste encore à prouver. Le réservoir est hypothétique et sa détermination, peu importante pour les zones d'endémie, est essentielle pour les pays indemnes. Une enquête sérologique récente faite en fixation du complément chez 1500 chevaux sénégalais, révèle que 60 p. 100 des chevaux vivant en zone d'élevage intensif ou en zone sylvo-pastorale, ont eu un contact récent avec le virus. On peut penser que cette zone représente une niche écologique où le virus se maintient dans son cycle fondamental.
\end{abstract}

Le virus de la P.E.A., d'après les travaux de OLLERMAN, ELS et ERASMUS (1970) est classé maintenant au même titre que le virus de la «Bluetongue » dans un sous-groupe des diplornavirus, mais l'épidémiologie de la maladie l'apparente à celle des arbovirus. Ceci suppose l'intervention d'un vecteur appartenant aux arthropodes hématophages et l'existence d'un hôte naturel ou réservoir. Le cheval, quant à lui, joue le rôle d'un hôte accidentel pouvant dans certaines conditions constituer pour le virus une impasse biologique.

Jusqu'ici, la preuve expérimentale de la transmission par un vecteur selon les critères de l'O.M.S. n'est pas encore faite et l'existence du réservoir est hypothétique. Or, comme le souligne Mc INTOSH (10), si l'identification du réservoir est sans importance pour les contrées où la maladie est endémique, elle est primordiale pour les pays neufs ou menacés.

(*) I.E.M.V.T., Laboratoire National de l'Elevage, B.P. 2057, Dakar-Hann, République du Sénégal.
Dans cet exposé, nous analyserons brièvement les principaux faits et travaux concernant l'écologie pour discuter trois questions majeures :

- l'apparition d'une épizootie,

- le vecteur,

- le réservoir.

\section{ETUDE ET ANALYSE DES FAITS}

\section{$1^{\text {er }}$ point : La répartition géographique}

Le berceau de la maladie est l'Afrique tropicale, l'Ile de Madagascar en étant exclue. Sur le continent africain, on trouve la P.E.A. de part et d'autre de l'équateur dans les pays de climat tropical sec dont le type est le climat sahélo-soudanais.

Périodiquement, la P.E.A. sort de son berceau originel pour toucher des pays parfois éloignés. 
Les flambées de la P.E.A. sont connues en Afrique australe depuis le $\mathrm{XVII}^{\mathrm{e}}$ siècle et principalement en Union Sud africaine où la maladie revient environ tous les 20 ans dans la région du Cap.

En Afrique septentrionale, ces flambées sont beaucoup moins régulières. En 1930-31, on trouve la P.E.A. en Egypte. En 1943-44, on l'observe de nouveau en Egypte, en Palestine et même au Liban. En 1959, l'Iran est touché puis vers l'Est, elle s'étend à l'Afghanistan, au Pakistan et à l'Inde (1960); vers l'Ouest, elle est reconnue à cette époque, en Irak, Jordanie, Syrie, Turquie, Liban et Chypre.

En 1965, elle franchit la barrière saharienne, touche le Sud marocain et le Sud algérien, puis après une accalmie pendant l'hiver, elle passe en 1966 aux régions Nords de ces deux pays et s'étend en Tunisie. En octobre 1966, elle touche le Sud de l'Espagne. Depuis, Ia maladie s'est retirée dans sa zone d'origine où elle demeurera jusqu'à la prochaine flambée sous la forme endémique.

\section{$2^{\text {e }}$ point : Aspect et diffusion de la P.E.A.}

Dans les pays africains à climat tropical sec, la maladie existe à l'état endémique. Un équilibre écologique s'établit entre le vecteur, le réservoir et les chevaux. Prenons l'exemple des pays d'Afrique occidentale et centrale situés au Nord de l'équateur entre les isohyètes $250 \mathrm{~mm}$ et $1000 \mathrm{~mm}$. Ces deux isohyètes délimitent une étroite bande traversant l'Afrique de part en part correspondant au climat tropical sec. Cette bande est favorable à l'élevage du cheval, on y dénombre près de 1500000 équins, la diffusion de cette espèce étant limitée au Nord par la proximité du désert et au Sud par la mouche tsé-tsé. L'introduction des chevaux au Sud du Sahara remonte d'après l'inventaire des rupestres sahariens au deuxième millénaire avant notre ère. Il est vraisemblable que ces animaux, en raison des contacts fréquents avec le virus P.E.A., ont acquis peu à peu l'état de résistance naturelle qui est propre aux races locales actuelles. La preuve du contact avec le virus est fournie par les enquêtes sérologiques, réalisées au Tchad par MAURICE et PROVOST (9) au Sénégal et pour une moindre part au Mali par BOURDIN, LAURENT, BERNARD et MBAYE (1966-1972).

\section{$3^{\mathrm{e}}$ point : Le climat et Ia topographie}

Tous les auteurs soulignent l'influence favorable d'une tension élevée de vapeur d'eau consécutive à des pluies abondantes, associées à la chaleur. Le froid et la sécheresse ont au contraire une action défavorable.

$\mathrm{Du}$ point de vue topographique, les auteurs reconnaissent aussi que la maladie est localisée de préférence dans les zones humides, marécages, vallées et près des points d'eau, puits et forages. En Afrique du Sud, on l'observe rarement au-dessus de 500 mètres sauf dans des conditions climatiques exceptionnelles.

\section{$4^{\text {e }}$ point : La réceptivité des animaux}

Le cheval possède une sensibilité aiguë. Dans les effectifs neufs, d'après RAFYI (23), 95 p. 100 des animaux sont touchés. Le mulet est moins sensible $(50$ p. 100 des sujets sont atteints). L'âne est insensible. Cependant, ALEXANDER (1), en Egypte, SERS en Algérie (26), ORUE (16) au Sénégal, ont observé des cas chez cette espèce.

A mentionner aussi les cas relevés parmi les chiens de meute nourris avec de la viande de cheval malade, observés par THEILER (29) et PIERCY (21).

\section{$5^{\circ}$ point : La transmission du virus}

$\mathrm{La}$ transmission directe du virus est exceptionnelle, il est possible de faire vivre des animaux neufs et des malades dans une écurie désinsectisée et protégée par des moustiquaires. Le mode de transmission est essentiellement indirect, il se fait par l'intermédiaire d'arthropodes hématophages.

\section{ETUDE ET ANALYSE DES TRAVAUX EXPERIMENTAUX}

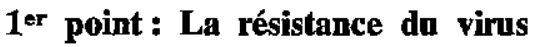

Le virus est thermo-stable, il résiste également à la dessiccation, mais il est sensible à des $\mathrm{pH}$ légèrement acides.

\section{$2^{e}$ point : La persistance du virus chez les animaux sensibles}

La présence du virus chez les animaux malades et notamment le cheval est de courte durée. 
Neuf jours après le début de la maladie, l'isolement du virus à partir du sang est rare. Mc INTOSH (10) pense que cet isolement est rendu difficile par la présence d'anticorps neutralisants qui masquent le virus. Cet auteur est parvenu à dissocier le virus P.E.A. masqué, par un transfert chez le furet inoculé par voie intracardiaque.

\section{3e point : Les vecteurs}

Les auteurs retiennent de nombreux arthropodes. JOUBERT (8) en a dressé la liste, mais il estime que beaucoup d'espèces ne jouent le rôle que de simples aiguilles ailées.

A l'heure actuelle, les principales espèces incriminées appartiennent au Culicidae, (moustiques) et au Ceratopogonidae (culicoïdes).

Si l'on examine les travaux des différents auteurs attachés à cette question, on remarque une certaine divergence dans les résultats. Pour NIESCHULZ, BEDFORD et DU TOIT (13), les moustiques du genre Aedes infectés sur chevaux malades transmettent exceptionnellement la maladie à des chevaux neufs. Pour OZAWA et ses collaborateurs $(18,19)$, il est possible de transmettre le virus à des chevaux sensibles à partir d'Anopheles stephensi, Culex pipiens fatigans, et Aedes aegypti nourris in vitro avec des suspensions de liquides virulents.

DU TOIT (6) trouve le virus chez les culicoïdes sans spécification d'espèce et réussit en 1969 la transmission, par l'intermédiaire de cet arthropode, d'un animal malade à un sujet sensible.

Récemment, WETZEL, NEVILL et ERASMUS (30) ne réussissent pas à isoler le virus P.E.A. à partir d'Aedes aegypti, de Culex pipiens fatigans ou de culicoides spp. nourris in vitro sur des liquides virulents, ou in vivo sur chevaux.

Ils échouent également au cours d'essais de transmissions avec les culicoïdes.

\section{0 point : Le réservoir, sa recherche et son identification}

Pour cette étude, on dispose de deux moyens, l'enquête sérologique et les essais d'isolement du virus.

L'enquête sérologique décèle les espèces susceptibles d'héberger ou d'avoir eu un contact avec le virus et ne peut être valable que si le nombre d'échantillons est suffisant. Il n'existe pas à notre connaissance d'enquête sérologique à grande échelle. Examinons les résultats des enquêtes relevés dans la littérature, PILOMORON (22) et ses collaborateurs, chez les ânes des oasis du Sud algérien, trouvent 69 p. 100 de résultats positifs sur 84 échantillons. Chez les chiens, Mc INTOSH (10) trouve des anticorps dans 1 sérum sur 13. SHAH (27) aux Indes trouve des anticorps chez le chien (10 sérums positifs sur 20 ), chez l'âne ( 8 sérums positifs sur 42) mais ne trouve pas trace d'anticorps dans des sérums d'oiseaux, de chèvres et de moutons.

Les essais d'isolement du virus par inoculation au souriceau de prélèvements issus d'animaux autres que les chevaux ont toujours été négatifs.

Cette grande difficulté à isoler le virus P.E.A. soit à partir des vecteurs, soit à partir de l'hôte, est soulignée par les études des chercheurs de l'O.M.S. sur les arbovirus.

Au Sénégal, ROBIN et BRES (24), ROBIN et LE GONIDEC (25), TAUFLIEB, CORNET et CAMICAS (28), ont inoculé 9788 prélèvements provenant de l'homme, d'animaux sauvages et d'arthropodes hématophages. Ils ont isolé 224 virus, mais aucun virus P.E.A. n'a encore été trouvé à ce jour. Au Nigéria, les arbovirologistes de l'O.M.S. (2) enquêtant sur place et dans les pays voisins, Dahomey, Cameroun, Tchad et Togo, ont inoculé 39696 prélèvements et isolé 1613 virus, aucun virus P.E.A. ne figure parmi eux. Pourtant, à plusieurs reprises, Ies chercheurs œuvrant au Nigéria ont isolé le virus de la bluetongue chez des culicoïdes, or, ce virus appartient au même groupe que le virus P.E.A.

\section{DISCUSSION}

Examinons à présent les conclusions que l'on peut extraire de ces analyses sur les problèmes de l'apparition d'une épizootie, du vecteur et du réservoir.

\section{L'apparition d'une épizootie}

Si l'on se réfère à l'épidémiologie des arboviroses, on peut concevoir que la P.E.A. se trans- 
met comme les arbovirus selon plusieurs cycles, BRES et ROBIN (5).

Dans un pays vierge comme l'Afrique avant l'introduction des chevaux, on peut imaginer l'existence d'un cycle fondamental selvatique, évoluant en l'absence du cheval et dont le diagramme est :

Vertébré infecté $\rightarrow$ arthropodes vecteurs $\rightarrow$ vertébré neuf...

Le cheval infecté peut être introduit dans une zone où vit une population équine réceptive à une saison où les anthropodes hématophages sont nombreux et les conditions écologiques favorables. La P.E.A. devient alors épizootique et le diagramme est :

Cheval infecté $\rightarrow$ arthropodes hématophages $\rightarrow$ cheval neuf.

Dans cette zone, les conditions écologiques peuvent varier fondamentalement selon l'époque de l'année et présenter des différences marquantes avec celles des pays à endémie permanente. Notamment retour au réservoir exclu, conditions climatiques plus tranchées interrompant brutalement la multiplication des vecteurs. Privée alors de son support endémique, la maladie s'éteint toute seule, soit au cours de l'année, ou l'année suivante ou même à l'extrême rigueur la troisième année.

\section{Le vecteur}

Le contexte écologique est en faveur d'une transmission par arthropodes hématophages, mais les preuves expérimentales sont encore contradictoires. Pourtant, deux groupes d'arthropodes sont retenus par les auteurs comme vecteurs probables, les culicoïdes et à un moindre titre les moustiques. Pour quel motif écologique est-il difficile de prouver expérimentalement cette transmission?

La première raison est d'ordre statistique. En Afrique du Sud, il est difficile d'isoler des culicoïdes de virus de la P.E.A. tandis que son cousin le virus de la bluetongue est facilement isolé de ce même groupe d'arthropodes. Cette différence peut provenir dans le rapport existant entre le nombre des hôtes. En effet, le virus de la bluetongue a à sa disposition 12800000 bovins hôtes réservoirs et
39000000 de moutons hôtes sensibles contre 380000 chevaux pour le virus de la P.E.A., seuls hôtes à partir desquels il est possible d'isoler le virus.

Le deuxième motif découle de la biologie des culicoïdes. Certaines espèces comme Culicoides pallidipennis vivent en rapport étroit avec le bétail et se reproduisent selon NEVILL (12) dans les excréments frais des bovins, mais le reste de leur biologie est mal connue, on sait toujours d'après NEVILL (12) qu'ils sont autogènes au premier cycle, autrement dit ils n'ont pas besoin d'un repas sanguin pour la première ponte et que, s'il est possible de distinguer les culicoïdes ayant eu un cycle gonotrophique, la numération des cycles est impossible.

Quant aux moustiques, leur biologie est beaucoup mieux connue. Malheureusement, dans le cas de la P.E.A., si la transmission expérimentale réussit avec certaines espèces, l'isolement du virus n'a pu être réalisé dans la nature. Ce qui fait des moustiques des vecteurs suspects, mais non confirmés. On peut expliquer la présence du virus en Iran et en Algérie pendant deux années successives par la persistance du virus chez certaines espèces pendant leur hibernation; il reste à le prouver.

Disons, pour conclure ce problème du vecteur, que pour beaucoup de virus transmis par les arthropodes et parmi eux 1a P.E.A., les critères fixés par les arbovirologistes sont loin d'être tous satisfaits.

\section{Le réservoir}

Le problème posé par le réservoir est loin d'être résolu. Le réservoir reste hypothétique et selon l'expression de MORNET et GILBERT (11), il doit être considéré pour le moment comme un «être de raison». Pourtant, sa mise en évidence est d'une grande importance pour les pays indemnes de P.E.A. comme le souligne Mc INTOSH (10).

Il est superflu de citer le nom des diverses espèces animales suspectes d'être le réservoir. Il est préférable d'envisager un plan de recherche calqué sur ceux habituellement appliqués à l'étude épidémiologique des arbovirus. Le point de départ de ce planning est une enquête sérologique chez les chevaux afin de localiser 
les zones à forte endémie où, les contacts entre virus et équins étant fréquents, la maladie existe sous la «forme rurale ». Ces contrats impliquent que le vecteur et le réservoir soient présents. Autrement dit, cette enquête sérologique doit permettre de localiser la niche écologique où le virus est maintenu dans son cycle fondamental.

Au Sénégal, BOURDIN, LAURENT, BERNARD et MBAYE ont examiné en fixation du complément 1500 sérums de chevaux provenant de deux zones. La zone agricole et la zone pastorale. La fixation du complément révèle que 20 p. 100 des chevaux de la zone agricole ont eu un contact récent avec le virus contre 66 p. 100 pour ceux vivant en zone pastorale.
La zone agricole est largement défrichée, mais dans la zone pastorale, l'élevage se fait selon le mode extensif, l'alimentation étant assurée par des pâturages naturels et l'abreuvement se fait soit au puits, soit à partir de forages. Autour des forages, vient entre 15000 et 20000 animaux : bovins, moutons, chèvres, chevaux, ânes, quelques dromadaires et des chiens de berger. En outre, la faune sauvage est représentée par de nombreux oiseaux, des hyènes, des chacals et de nombreuses autres espèces.

Aux abords des forages et des puits, on trouve l'eau stagnante et les excréments frais favorables à la reproduction des vecteurs. Il est très probable que, compte tenu des conditions écologiques, le réservoir est présent à proximité.

\section{SUMMARY}

\section{Note about ecology of african horse-sickness}

The analysis of facts and experimental works concerning the ecology of African Horsesickness (AHS), shows that this disease presents an endemic character in countries with dry tropical climate. The affection spreads periodically into the neighbouring countries situated north and south of this zone. Horses are very sensitive to the virus, but in territories where the disease occurs since a long time they acquired a natural and solid immunity.

The mode of propagation is similar to the transmission of arboviruses. Two vectors are involved mosquitoes and Culicoïdes. In nature the isolation of the virus is difficult and has only been achieved from. Culicoides sp. The multiplication of virus in suspected vectors remains to be proved. The reservoir is hypothetical and its determination presents little consequence in endemic areas; however, this determination is essential for free areas. A recent serological survey (CFT) carried out in Senegalese horses living in a zone of extensive cattle breeding (sylvo pastoral zone) shows that 60 p. 100 of the animal demonstrate a recent contact with the virus.

The authors suggest that sylvo pastorale zone can be considered as an ecological niche where the existence of the virus is maintained through a basic cycle.

\section{RESUMEN}

Nota sobre la ecologia de la peste equina africana (P.E.A.)

Segùn la analisis de los trabajos sobre la peste equina africana, se encuentra dicha enfermedad al estado endemico en los paises con un clima tropical seco y sobrepasa al sur y al norte hacia los paises vecinos. El caballo es sensible al virus pero, en los paises donde la P.E.A. es antigua, ha adquirido una inmunidad natural sólida. El modo de transmisión se emparenta al de los arbovirus; se incriminan dos vectores: los mosquitos y los Culicoides spp. La multiplicación del virus en los dos vectores todavia queda por probar.

El reservorio es hipotético y su determinación poco importante para las zonas de endemia es esencial para los paises indemnes. Una encuesta serologica reciente utilizando la fijación del complemento en 1500 caballos de Senegal muestra que 60 p. 100 de los caballos viviendo en zona de cria intensiva $o$ en zona silvopastoral, tuvieron un contacto reciente con el virus. Se puede pensar que esta zona representa un sitio ecologico dónde se mantiene el virus en su ciclo fundamental. 


\section{BIBLIOGRAPHIE}

1. ALEXANDER (R. A.). The 1944 epizooty of horse sickness in the Middle East. Onderstepoort J. vet. Sci. anim. Ind., 1948, 23 : 77-92.

2. Arbovirus Research Project. Animal report. University of Ibadan. 1964-65-66-67-68-69.

3. BOURDIN (P.), BERNARD (G.), LAURENT (A.) et MBAYE (A.). Enquête sérologique sur la peste équine au Sénégal (en préparation).

4. BOURDIN (P.). Non publié (1966).

5. BRES (P.) et ROBIN (Y.). Les arbovirus : généralités. Dengue. Fièvre à phlébotome. Encycl. Méd. chir. Mal. Inf., 1970, 8065 E 10: 1-12.

6. DU TOIT (R.M.). Transmission of bluetongue and horse sickness by $C_{\text {ullicoides. Onderstepoort }}$ J. vet. Sci. anim. Ind., 1944, 19:7-16.

7. DU TOIT (R.M.). Cité par Wetzel, Nevill et Erasmus, 1970.

8. JOUBERT (L.). Alerte à la peste équine. L'épizootie au Maroc et la protection en France. Rec. Méd. vét., 1967, 118 : 49-57.

9. MAURICE (Y.) et PROVOST (A.). La peste équine à type 9 en Afrique centrale. Enquête sérologique. Rev. Elev. Méd. vét. Pays trop., 1967, 20: $21-25$

10. Mc INTOSH (B. M.). Immunological types of horse sickness virus and their significance in immunization. Onderstepoort J. vet. Res., 1958, 27 : 465-538.

11. MORNET (P.) et GILBERT (Y.). La peste équine. Paris, l'Expansion, 1968 (Coll. Les maladies animales à virus).

12. NEVILL (E. M.). A significant new breeding site of Culicoides pallidipennis Carter Ingram and Nacfie (Dipters: Caratopogonides). J.S. Afr. vet. Med. Ass., 1968, 39: 61.

13. NIESCHULZ (O.), BEDFORD (C. A. M.) et DU TOIT (R.). Investigation into the transmission of horse sickness at Onderstepoort during the season 1931-32. Onderstepoort J. vet. Sci., 1939, $3: 275$ 334.

14. NIESCHULZ (O.) et DU TOIT (R.). Investigation into the transmission of horse sickness at Onderstepoort during the season 1932-33. Onderstepoort J. vet. Sci., 1937, $8: 213-270$.

15. OLLERMANN (R. A.), ELS (H. J.) et ERASMUS (B. J.). Characterization of African horse sickness virus. Arch. ges. Virus forsch., 1970, 29, 163-174.

16. ORUE (J.). Communication personnelle. 1972.

17. OZAWA (Y.), NAKATA et HAZRATI (A.). Growth of african horse sickness virus in monkey kidney cell culture. Amer. J. vet. Res., 1964, 25 : 505-511.

18. OZAWA (Y.), NAKATA (G.). Experimental transmission of african horse sickness by means of mosquitoes. Am. J. vet. Res., 1965, 26 : 744748.

19. OZAWA (Y.), NAKATA (G.) et SHAD DEL (F.). Experimental transmission of african horse sickness by means of mosquitoes. Arch. Inst. Razi., 1966, 18 : 119-125.

20. OZAWA (Y.), SHAD DEL (F.), NAKATA (G.) et NAVAI (S.). Transmission of african horse sickness by means of mosquito bites and replication of the virus in Aedes aegypti. Arch. Inst. Razi., 1970, 22 : 113-122.

21. PIERCY (S. E.). Some observations on african horse sickness including an account of an outbreak amongst dogs. East. Afr. Agric. J., 1951, 17, 62-64.

22. PILO-MORON (E.), VINCENT (J.), AIT MESBAN (O.) et FORTHOMME (G.). Origine de la peste équine en Afrique du Nord : résultats d'une enquête sur les ânes du Sahara algérien, Arch. Inst. Pasteur Algérie, 1969, 47: 105-118.

23. RAFYI (A.). La peste équine. Bull. off. int. Epiz., 1961, 56: 170-215.

24. ROBIN (Y.) et BRES (P.). Recherches effectuées sur l'écologie des arbovirus au Sénégal. Projet O.M.S. - Rapport annuel Institut Pasteur, Dakar, 1965-66-67-68-69.

25. ROBIN (Y.), LE GONIDEC (C.). Recherches effectuées sur l'écologie des arbovirus au Sénégal. Rapport annuel. Projet O.M.S. 1970.

26. SERS (J, L.). La peste équine en Algérie. Etude sur l'épizootie d'automne 1965. Thèse Méd. vét. Alfort, 1967, $\mathrm{n}^{4} 4$.

27. SHAH (K. V.). Investigation of african horse sickness in India. I. Study of the natural disease and the virus. Indian $J$. vet. Sci., 1964, 34:1-14.

28. TAUFFLIEB (R.), CORNET (M.) et CAMICAS (J. L.). Recherches effectuées sur l'écologie des arbovirus au Sénégal. Projet O.M.S. - Rapport annuel O.R.S.T.O.M. Dakar, 1965-66-67-68-69-70.

29. THEILER (A.). The susceptibility of the dog to african horse sickness. J. comp. Path. Ther, 1910, 23: $315-325$.

30. WETZEL (H.), NEVILL (E. M.) et ERASMUS (B. J.). Studies on the transmission of african horse sickness, Onderstepoort J. vet. Res., 1970, 37 : $165-168$. 